\title{
The CALICE Digital Hadron Calorimeter: Calibration and Response to Pions and Positrons
}

\section{Burak Bilki ${ }^{* \dagger}$}

Argonne National Laboratory, Argonne, IL 60439 USA

University of Iowa, Iowa City, IA 52242 USA

E-mail: burak-bilki@uiowa.edu

\begin{abstract}
The large CALICE Digital Hadron Calorimeter prototype (DHCAL) was built in 2009 - 2010 and was tested in the Fermilab and CERN test beams. The DHCAL uses Resistive Plate Chambers (RPCs) as active media and is read out with 1 x $1 \mathrm{~cm}^{2}$ pads and digital (or 1 - bit) resolution. With a world record of nearly $480 \mathrm{k}$ readout channels, the DHCAL offers the possibility to study hadronic interactions with unprecedented spatial resolution. Here we report on the results from the analysis of pion and positron events of momenta between 2 to $60 \mathrm{GeV} / \mathrm{c}$ collected in the Fermilab test beam. Particular emphasis is given to the intricate calibration procedure. The analysis demonstrates the unique utilization of detailed event topologies.
\end{abstract}

Technology and Instrumentation in Particle Physics 2014,

2-6 June, 2014

Amsterdam, the Netherlands

\footnotetext{
* Speaker.

${ }^{\dagger}$ On behalf of the CALICE Collaboration
} 


\section{Introduction}

A novel approach, named Particle Flow Algorithms (PFAs) is proposed to achieve unprecedented jet energy resolution of the order of 3-4 \% at a future linear collider [1]. The CALICE Collaboration develops calorimeters that are optimized for the application of PFAs. The large CALICE Digital Hadron Calorimeter (DHCAL) prototype was built in 2009-2010 with a design based on the preliminary work done with a small-scale prototype, which underwent a rigorous test program in the Fermilab test beam and resulted in numerous publications [2]. The active media of the DHCAL are Resistive Plate Chambers (RPCs), which are read out by $1 \times 1 \mathrm{~cm}^{2}$ pads with a 1-bit resolution (digital readout). A single layer of the DHCAL measures roughly $1 \times 1 \mathrm{~m}^{2}$ and consists of 96 x 96 pads. During the Fermilab beam tests, the calorimeter consisted of a 38-layer structure (main stack) with $1.75 \mathrm{~cm}$ thick steel absorber plates and a 14-layer structure (tail catcher) with eight $2 \mathrm{~cm}$ thick steel plates followed by six $10 \mathrm{~cm}$ thick steel plates. In addition to the absorber plates, each layer of RPCs was contained in a cassette with a $2 \mathrm{~mm}$ thick Copper front plate and a $2 \mathrm{~mm}$ thick Steel back plate. The details of the DHCAL can be found in $[3,4]$.

The DHCAL is a calorimeter with the following unique features: RPCs for calorimetry (no other hadron calorimeter uses RPCs as active medium); pad readout of RPCs (RPCs are usually read out with strips); digital readout; embedded front-end electronics; large channel count (a world record of $0.5 \mathrm{M}$ channels). Here, we briefly describe the basics of the intricate calibration procedures and their implementation in the analysis of the Fermilab data.

\section{Calibration Procedures}

The DHCAL data contain the hit position information, the time stamp of the individual hits and the time stamp from the trigger and timing unit. Additionally, discriminated signals from a beam Čerenkov counter and a muon tagger are integrated into the data stream by the data acquisition system. The hits in each layer are combined into clusters using a nearest-neighbor algorithm.

The calibration of the DHCAL starts with measuring the performance parameters of the individual RPCs, i.e. the efficiency and the average pad multiplicity. The purpose of the calibration procedures is to minimize the variations in the response of the DHCAL resulting from the variations in these performance parameters. Here two methods are used: track fits and track segment fits. In order to avoid a bias in the estimation of the performance parameters, regions within $1 \mathrm{~cm}$ of dead/hot cells or RPC edges are excluded from these measurements.

The track fits method uses dedicated muon calibration runs to assess the performance parameters of individual RPCs. This method starts with grouping the clusters that are laterally within a distance of $3 \mathrm{~cm}$ of each other in different layers for the events with tracks traversing the DHCAL. The group of clusters, excluding clusters belonging to the layer being investigated, is then fit to a 3-dimensional parametric line. For the layer being investigated, clusters within $2 \mathrm{~cm}$ of the point predicted by the fit are searched for. If a cluster is found, the layer is counted as efficient, and inefficient otherwise. If the layer is efficient, the pad multiplicity is given by the size of the found cluster.

The track segment fits method is developed to measure the calibration parameters using the track segments within hadronic showers. With this method, the DHCAL provides another unique 
feature in calorimetry: For operation in a colliding beam environment, the DHCAL does not need a dedicated calibration system, as track segments can be used to monitor the performance of the RPCs. The method starts with searching for four clusters that are aligned within $3 \mathrm{~cm}$ in four layers surrounding the layer being investigated. The track segment is then fit to the parametric line defined above and this track segment is used to measure the performance parameters of a fifth layer.

Using the track fits and track segment fits methods, the calibration factors per RPC per data taking run are obtained as $\mathrm{C}_{i}=\varepsilon_{i} \mu_{i} / \varepsilon_{0} \mu_{0}$ where $\varepsilon_{i}$ and $\mu_{i}$ are the efficiency and the average pad multiplicity of RPC i and $\varepsilon_{0}$ and $\mu_{0}$ are the average RPC efficiency and pad multiplicity of the entire stack, 0.96 and 1.56 respectively [4].

In the second step of the calibration, the number of hits measured in a given RPC is corrected for differences in its performance parameters using three different approaches: full calibration, density-weighted calibration, and hybrid calibration.

Full Calibration: The hits in RPC i are weighted by $1 / C_{i}$.

Density-Weighted Calibration: This approach takes into account that pads collecting charge from several nearby avalanches, for instance in the core of a shower, require a different calibration procedure than pads measuring single tracks. In other words, a pad in the core of a shower will register a hit with minimal dependence on the performance characteristics of this particular RPC and it should be calibrated in a different way than a pad e.g. along a MIP track in the same RPC. In this approach, the calibration factors of a pad depend on the local hit density (as an estimate of the number of avalanches contributing to the signal charge of that pad), the energy of the incident particle, and the type of incident particle, in addition to the performance parameters of the RPCs.

Hybrid Calibration: For the hits with 0 or 1 neighbor, the density effect is minimal. The hybrid calibration utilizes full calibration for density bins 0 and 1 , and density-weighted calibration for the higher density bins.

Further details about the calibration approaches can be found in [5].

\section{Results}

As an example of the implementation of the calibration procedures, Fig. 1 shows the mean response (left) and the energy resolution (right) for the uncorrected pion data (black) and densityweighted calibration (green). The mean response is fit to the power function $\mathrm{N}=\mathrm{aE}^{m}$ up to and including $60 \mathrm{GeV}$. The resolutions are fit to a generic resolution function $\frac{\sigma(N)}{N}=\frac{\alpha}{\sqrt{E}} \oplus C$ where $\alpha$ is the stochastic term and $\mathrm{C}$ is the constant term. The resolution fits are up to and including the $25 \mathrm{GeV}$ point and they are extrapolated to $60 \mathrm{GeV}$. The details of the event selection and the topological particle identification are given in [5]. All calibration schemes tend to normalize the mean response to the predefined DHCAL operating conditions [5]. At lower energies, the methods agree with each other. However, at higher energies where the shower densities are large, the effect of employing the density weighting in the calibration procedure is noticable [5].

\section{Summary}

The DHCAL recorded around 14 million secondary beam events over five test beam campaigns at Fermilab. The beam is a momentum-selected mixture of muons, pions and positrons. Data 

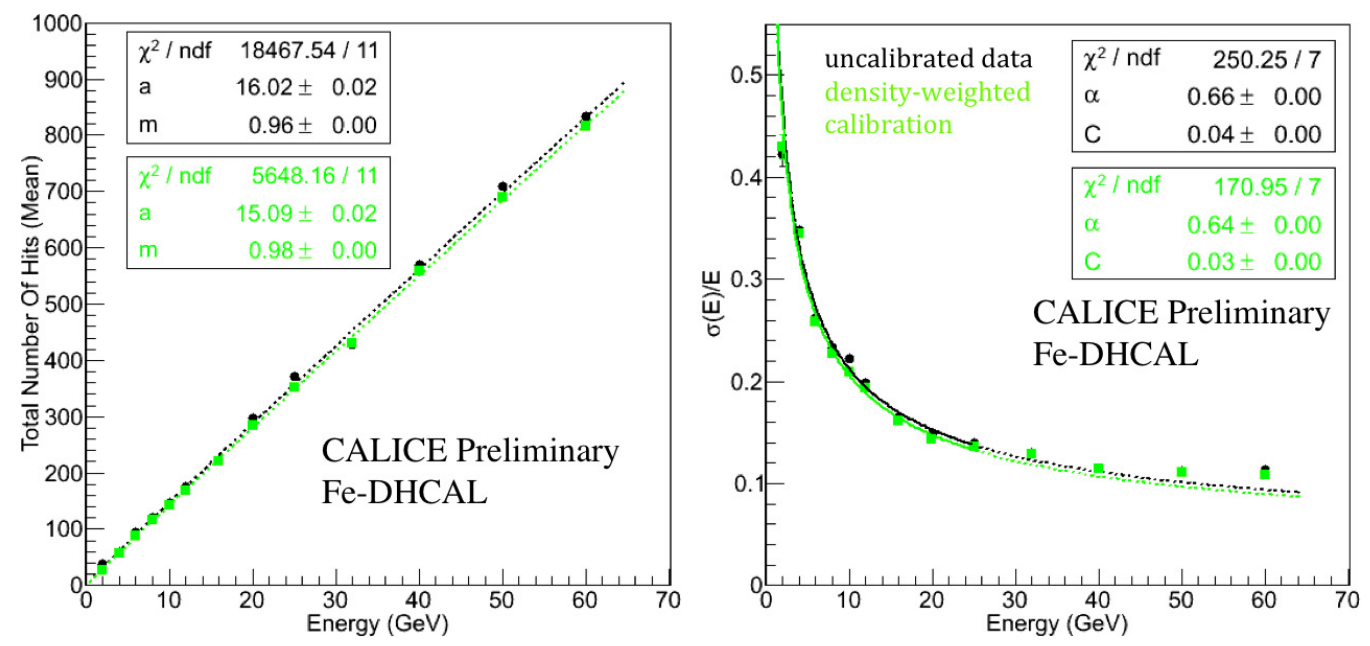

Figure 1: Mean response (left) and resolution (right) for the uncalibrated pion data (black) and the densityweighted calibration (green).

were collected at various energies between 2 and $60 \mathrm{GeV}$. The high granularity and the digital readout of the DHCAL enable the utilization of numerous topological event parameters for all purposes ranging from calibration to correcting the hadronic/electromagnetic response (software compensation) and improvements to the energy resolution measurements.

The calibration of the DHCAL is based on two performance parameters of the Resistive Plate Chambers: efficiency and average pad multiplicity. A simple multiplication of these parameters normalized to a reference value can serve as a calibration factor. However, the density of showering particles per pad impacts the calibration procedure in a complicated manner. As a result, the density-weighted calibration schemes provide better handles in understanding/manipulating response differences due to changes in individual RPC performances and operation conditions of the DHCAL.

All three calibration schemes, i.e. full calibration, density-weighted calibration and hybrid calibration, result in a more uniform response for all runs at each energy point when compared to the uncalibrated results.

\section{References}

[1] M.A. Thomson, Nucl. Instrum. Meth. A 611 25, 2009; J.C. Brient et al., eConf C 010630 E3047, 2001; V.L. Morgunov, Proc. of Calorimetry in Particle Physics (CALOR 2002), Pasadena USA, 2002.

[2] Q. Zhang et al., JINST 5 P02007, 2010; B. Bilki et al., JINST 4 P10008, 2009; B. Bilki et al., JINST 4 P06003, 2009; B. Bilki et al., JINST 4 P04006, 2009; B. Bilki et al., JINST 3 P05001, 2008; G. Drake et al., Nucl. Instrum. Meth. A 578 88, 2007.

[3] http://www.hep.anl.gov/repond/DHCAL_FNAL_Nov_2010.pptx

[4] J. Repond, CALICE Analysis Notes CAN-030, 2011 and CAN-030A, 2013.

[5] B. Bilki, The DHCAL Results from Fermilab Beam Tests: Calibration, CALICE Analysis Note CAN-042, 2013. 